\title{
Aggressive behaviour of anti-vaxxers and their toxic replies in English and Japanese
}

Kunihiro Miyazaki ( $\square$ kunihirom@acm.org )

The University of Tokyo

\section{Takayuki Uchiba}

Sugakubunka Co., Ltd

Kenji Tanaka

The University of Tokyo

Kazutoshi Sasahara

Tokyo Institute of Technology

\section{Research Article}

Keywords: Social Media, Anti-vaxxers, COVID-19, Reply, Social media, Toxicity

Posted Date: January 24th, 2022

DOI: https://doi.org/10.21203/rs.3.rs-1289044/v1

License: (c) (i) This work is licensed under a Creative Commons Attribution 4.0 International License.

Read Full License 


\title{
Aggressive behaviour of anti-vaxxers and their toxic replies in English and Japanese
}

\author{
Kunihiro Miyazaki ${ }^{*}$, Takayuki Uchiba ${ }^{2}$, Kenji Tanaka ${ }^{1}$ \\ and Kazutoshi Sasahara ${ }^{3^{*}}$ \\ ${ }^{1 *}$ The University of Tokyo, Tokyo, Japan. \\ ${ }^{2}$ Sugakubunka Co., Ltd., Tokyo, Japan. \\ ${ }^{3}$ Tokyo Institute of Technology, Tokyo, Japan.
}

\author{
*Corresponding author(s). E-mail(s): kunihirom@acm.org; \\ sasahara.k.aa@m.titech.ac.jp; \\ Contributing authors: takayuki.uchiba@sugakubunka.com; \\ tanaka@tmi.t.u-tokyo.ac.jp;
}

\begin{abstract}
The anti-vaccine movement has been gaining traction in many countries since the COVID-19 pandemic began. However, their aggressive behaviour in replies - the form of directed messaging that can be sent beyond follow-follower relationships-is still less understood, and even less so about their differences in languages. We conducted a comparative study of anti-vaxxers' aggressive behaviours by analysing a longitudinal dataset of COVID-19 tweets in English and Japanese. We found two common features across these languages. First, anti-vaxxers most actively transmit targeted messages or replies to users with different beliefs, especially to neutral accounts, with significantly toxic and negative languages; these replies are often directed to posts about vaccine operations. Second, the influential users with many followers and/or with titles are more likely to receive the most toxic replies from the anti-vaxxers. However, pro-vaccine accounts with a few followers even receive highly toxic replies in English, which calls for a special aid different from the Japanese case. These results provide insights into both language dependent and independent countermeasures against anti-vaxxers' aggressive behaviour.
\end{abstract}

Keywords: Social Media, Anti-vaxxers, COVID-19, Reply, Social media, Toxicity 


\section{Introduction}

The COVID-19 pandemic started at the end of December 2019 and spread rapidly around the world, affecting people both in the global health and economy. The pandemic also caused the overabundance and spread of misinformation related to COVID-19, such as incorrect treatments, conspiracy theories, and pseudoscience about vaccines [1] which induced secondary damage to society. In particular, the vaccine hesitancy caused by anti-vaxxers with misinformation and anxiety-provoking information is an urgent social problem, as it may delay or hinder the widespread of vaccination [2] and the achievement of herd immunity toward a post-pandemic world [3]. The World Health Organization (WHO) has called such a flood of mis/disinformation under pandemic as an 'infodemic' . To solve this problem, it is necessary for us to have a scientific understanding of anti-vaccine behaviour online and develop effective countermeasures across countries.

Much research has focused on the social media ecology of anti-vaxxers, i.e., anti-vaccine advocates; their thoughts and claim contents [4, 5]; behavioural patterns, emotions, topics, and positions in the social networks [6]; changes of their attitudes over time [7]; temporal patterns of how fake information spreads [8]; and motivations for sharing misinformation [9]. Although many attempts have been made in these directions, we have yet to know the most direct messaging form for anti-vaxxers' beliefs on social media, that is, reply behaviour. Unlike other forms of messaging behaviours (e.g. posts, shares), replies can be sent beyond the boundaries of follow-follower relationships, thereby gaining many impressions ${ }^{2}$ of the targets and their followers. Especially on vaccine topics, users are often inside 'echo chambers' with the belief and they only see what they want to see [10], but in reality, a reply can reach anyone regardless of the follow-follower networks $[11]^{3}$. Furthermore, most of the existing studies on anti-vaxxers' behaviours have analysed English data, and it is unclear whether the findings from these studies are valid across different languages.

In this paper, we empirically examine characteristics of anti-vaxxers' reply behaviours in the context of COVID-19 infodemic by analysing a longitudinal dataset of tweets in English and Japanese (see Data collection). The ratio of replies among all tweets turned out to be considerably small; nevertheless, the reply behaviors of anti-vaxxers are indispensable to study because recipients may be seriously damaged when receiving a few negative replies from an unknown user outside of follow-follower networks. We found that in both languages, anti-vaxxers most frequently sent replies to clusters with different briefs, especially to neutral accounts, and the content of their replies was significantly toxic and emotional. Furthermore, the most-targeted users were conspicuous accounts with large numbers of followers, including accounts

\footnotetext{
${ }^{1}$ https://www.who.int/health-topics/infodemic

${ }^{2}$ https://en.wikipedia.org/wiki/Impression_(online_media)

${ }^{3}$ Twitter recently added a feature that allows users limit accounts that can reply to them, but most users still permit free reply access to them. https://techcrunch.com/2020/08/11/twitter-no w-lets-everyone-limit-replies-to-their-tweets /
} 

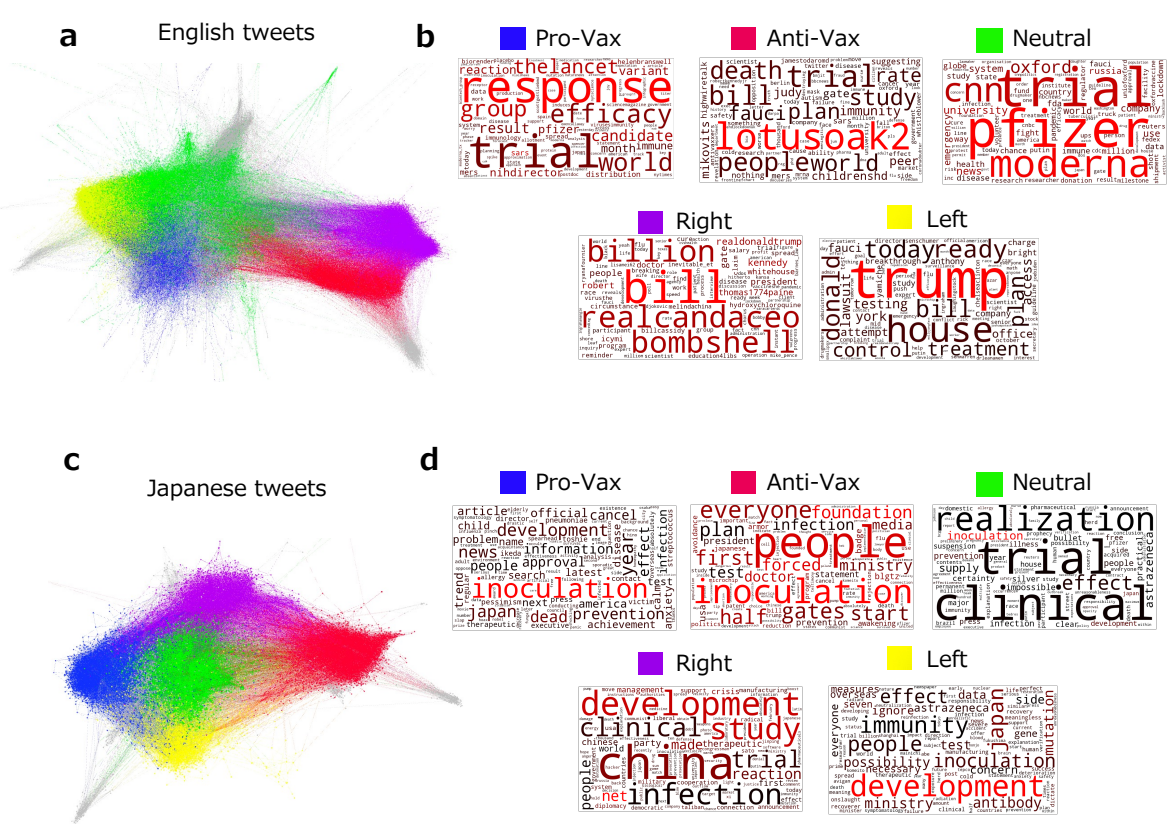

Fig. 1 RT network (a) for English users and the corresponding word clouds (b) and Japanese counterparts (c, d). In (a), the number of nodes is 47,135 and the number of edges is 241,370 , while in (c), the number of nodes is 12,017 and the number of edges is 62,132 . Nodes represent users and links represent retweets, and colours correspond to clusters. The five biggest clusters were found in both networks: in (a), Pro-Vax (9.17\%), Left (Anti-Trump) (16.31\%), Neutral (24.84\%), Anti-Vax (12.23\%), and Right (Pro-Trump) (12.18\%); in (c), Pro-Vax (18.6\%), Left (16.02\%) Anti-Vax (19.5\%), Neutral (25.42\%), and Right (10.86\%). In the word clouds (b) and (d), font size is corresponding to keyword frequency and font colour is corresponding to their Tf-Idf values (i.e. word importance). In (d), Japanese keywords are translated into English.

related to healthcare or representing scientists, policymakers, or media figures or outlets. Based on the findings, we finally discuss possible countermeasures, which may be useful for pro-vaxxers, fact-checkers, and platformers, in a multilingual setting, to create guidelines and develop an automated system that detects harmful content, including the mis/disinformation of vaccines.

\section{Results}

\section{Communities related to vaccine discourse}

We constructed the retweet (RT) networks from the COVID-19 tweets to identify anti-vaxxers and other groups (see Methods). The resulting networks are illustrated in Fig. 1a for English and 1c for Japanese. Figs 1b and 1c show popular words used in each group.

We found five clusters or groups in English tweets (Fig. 1a). Although these groups commonly used factual words related to COVID-19 and vaccine ('trial', 'response'), they also used different keywords specific to each group 
(Fig. 1b). One group paid much attention to the efficacy and the evidence of vaccines ('efficacy', 'the lancet'), suggesting the Pro-Vax group. Another group focused on conspiracy theories and criticism for the government ('bill', 'fauci' ${ }^{4}$ ), suggesting the Anti-Vax cluster; especially, a large portion of conspiracies consisted of Bill Gates, one of the biggest proponents of vaccination ${ }^{6}$. Yet another group that mentioned the topics of Bill Gates's investment in vaccine and some political names ('bill', 'billion', 'kennedy', 'realdonaldtrump') seemed to be a political right cluster, whereas the one that contained words for politics and government ('trump', 'house') seemed to be a political left cluster. In addition, we identified one that contained vaccine makers, universities, and news media outlets ('cnn', 'oxford', 'pfizer', 'moderna') as a neutral cluster. We further confirmed the above observations by checking the most retweeted accounts and tweets for each group (see Supplementary Information (SI)). While in most clusters the popular accounts included various users, such as politicians, journalists, doctors, anonymous influencers, organizations (WHO, the White House), the neutral cluster was mainly occupied by news media such as CNN and Reuters (see SI).

The comparable five clusters with topic structures emerge in the Japanese dataset (Fig. 1c): the pro- and anti-vaxxers, the political Left and Right clusters, and the Neutral cluster. Besides factual words ('inoculation', 'development'), the Pro-Vax group used words related to the efficacy of vaccination ('prevention', 'effect'), whereas the Anti-Vax group used words related to conspiracy theories and criticism for the government ('gates', 'ministry'7). In the political clusters, the Right cluster criticized China ('china'), whereas the Left group criticized the government ('ministry'). In addition to Japanese news media, vaccine makers' accounts ('astrazeneca') and words related to the possibility of vaccination ('realization', 'clinical trial') were found in the neutral group. Thus, the vaccine information ecosystems show striking similarities in English and Japanese, although they have different vaccine policies and political backgrounds. This structural and topical resemblance suggests that vaccination is a common political matter across countries [12], and perhaps weaponised during the COVID-19 infodemic $[13,14]$.

\section{Active reply by anti-vaxxers}

After identifying all the groups, we analysed how actively anti-vaxxers targeted other groups using replies. Figs. 2a and 2c show that both in English and Japanese, anti-vaxxers were the most active in reply behaviour. When we look at the reply frequencies in inner-cluster (i.e. replies to the same cluster) and inter-cluster (i.e., replies to the other clusters) conditions, we find two similarities in both languages. While the majority of replies were directed towards the same cluster (inner-cluster), the Anti-Vax groups sent the largest amount

\footnotetext{
${ }^{4}$ Dr. Fauci ('fauchi'), the director of the NIAID ${ }^{5}$

${ }^{6}$ https://www.gatesfoundation.org/our-work/programs/global-health/vaccine-development-an d-surveillance

${ }^{7}$ The Ministry of Health, Labor and Welfare of the Japanese government
} 
a

\begin{tabular}{|l|r|r|r|r|r|} 
& User & TW & TW/User & RP & RP/TW(\%) \\
\hline Pro-Vax & 4,320 & 216,130 & 50.0 & 6,501 & $3.01 \%$ \\
\hline Right & 5,739 & 203,288 & 35.4 & 1,419 & $0.70 \%$ \\
\hline Anti-Vax & 5,766 & 282,665 & 49.0 & 14,291 & $5.06 \%$ \\
\hline Left & 7,689 & 351,436 & 45.7 & 3,302 & $0.94 \%$ \\
\hline Neutral & 11,713 & 544,882 & 46.5 & 6,800 & $1.25 \%$ \\
\hline
\end{tabular}

C

\begin{tabular}{|l|r|r|r|r|r|}
\hline & User & TW & TW/User & RP & \multicolumn{2}{|c|}{ RP/TW(\%) } \\
\hline Pro-Vax & 2,238 & 87,706 & 39.2 & 1,963 & $2.24 \%$ \\
\hline Right & 1,305 & 40,048 & 30.7 & 502 & $1.25 \%$ \\
\hline Anti-Vax & 2,339 & 116,289 & 49.7 & 3,306 & $2.84 \%$ \\
\hline Left & 1,925 & 90,346 & 46.9 & 2,527 & $2.80 \%$ \\
\hline Neutral & 3,055 & 125,965 & 41.2 & 1,227 & $0.97 \%$ \\
\hline
\end{tabular}

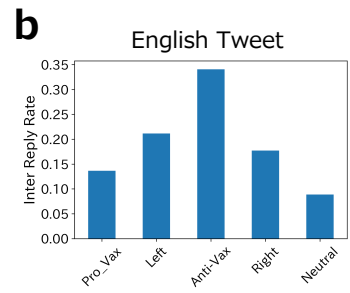

d

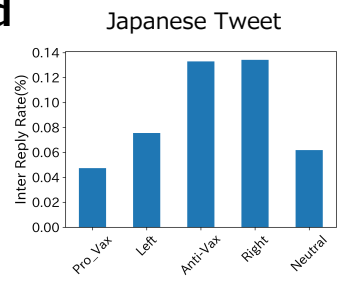

Fig. 2 Tweet and reply activities between clusters. a, Reply activities of each cluster in English tweets. Here, TW: tweets. RP: replies. TW includes normal tweets, RP, and RT. Reply rate (RP/TW) was significantly higher in the Anti-Vax cluster $\left(\chi^{2}\right.$ test: $p<0.001$, shown in rad shades); $\mathbf{b}$, Ratio of inter-cluster replies per all replies from each cluster in English tweets; $\mathbf{c}$ and $\mathbf{d}$ are the Japanese counterparts.

of inter-replies (n.b., the Right cluster is comparable in Japanese). Thus, antivaxxers are supposed to be more enthusiastic about reaching out to people with other beliefs. In contrast, pro-vaxxers sent out direct messages to external clusters at a lower frequency according to the inter-reply rate, although they should have played a role in disseminating the correct knowledge about the COVID-19 vaccination.

\section{Reply targets of anti-vaxxers}

Next, we examined the main targets of anti-vaxxers (Figs. 3a and 3d). Although all the groups were mainly targeting the Neutral group, the AntiVax groups had this tendency more apparent than others (shaded in red) in both languages (especially in English). This result is consistent with the findings of previous research that anti-vaxxers are more entangled with neutral groups than pro-vaxxers and thus successful in their reach [15]. On the contrary, the rate of replies from Anti-Vax to Pro-Vax is smaller than that of their opposite counterparts in both languages (shaded in yellow). This asymmetry in reply frequency suggests that the Anti-Vax group tends to neglect the Pro-Vax group.

Furthermore, we found that the reply targets have large numbers of followers. The median scores of followers of reply receivers are much larger than those of reply senders (see SI). To quantify the tendency of replies toward influential accounts, we calculated the percentage of replies directed to accounts with more than 10,000 followers (Figs. 3c and $3 \mathrm{f})^{8}$. We found that many of

\footnotetext{
${ }^{8}$ On Twitter, accounts with followers of more than 10,000 have much influence, and the number of 10,000 is an official milestone to be recognized as a large account https://www.cnbc.com/202 1/09/01/how-to- use-twitter-super-follows-to-earn-money.html
} 
a

\begin{tabular}{|l|r|r|r|r|r|r|}
\hline & \multicolumn{5}{|c|}{ Ratio among Reply Target (\%) } \\
\hline & Pro-Vax & Left & Anti-Vax & Right & Neutral \\
\hline Pro-Vax & - & 21.4 & 35.8 & 10.6 & 32.1 \\
\hline Left & 19.4 & -- & 4.6 & 37.9 & 38.1 \\
\hline Anti-Vax & 13.9 & 7.6 & -- & 23.5 & 55.0 \\
\hline Right & 10.1 & 13.8 & 41.3 & -- & 34.8 \\
\hline Neutral & 21.6 & 44.0 & 13.5 & 20.9 & -- \\
\hline
\end{tabular}

English Tweet

\section{b}

\begin{tabular}{|l|r|r|r|r|r|}
\hline & \multicolumn{5}{|c|}{ Ratio of Big Accounts (\%) } \\
\hline & Pro-Vax & Left & Anti-Vax & Right & Neutral \\
\hline E Pro-Vax & 56.9 & 90.1 & 23.3 & 98.5 & 93.9 \\
\hline L Left & 80.0 & 93.0 & 68.4 & 100.0 & 93.0 \\
\hline Anti-Vax & 38.3 & 92.8 & 61.7 & 97.5 & 94.7 \\
\hline Right & 42.9 & 100.0 & 73.7 & 96.5 & 91.7 \\
\hline Neutral & 78.7 & 94.3 & 52.5 & 95.6 & 90.2 \\
\hline
\end{tabular}

Japanese Tweet

d c

\begin{tabular}{|l|r|r|r|r|r|r|} 
& \multicolumn{5}{|c|}{ Ratio among Reply Target (\%) } \\
\hline & Pro-Vax & Left & Anti-Vax & Right & Neutral \\
\hline Pro-Vax & -- & 27.6 & 19.7 & 11.8 & 40.8 \\
\hline L Left & 18.7 & - & 32.9 & 11.0 & 37.4 \\
\hline Anti-Vax & 6.0 & 36.1 & -- & 13.2 & 44.7 \\
\hline Right & 25.5 & 2.1 & 27.7 & -- & 44.7 \\
\hline Neutral & 26.3 & 28.1 & 12.3 & 33.3 & - \\
\hline
\end{tabular}

\begin{tabular}{|l|r|r|r|r|r|}
\hline & \multicolumn{5}{|c|}{ Ratio of Large Accounts (\%) } \\
\hline & Pro-Vax & Left & Anti-Vax & Right & Neutral \\
\hline Pro-Vax & 32.1 & 52.4 & 33.3 & 77.8 & 64.5 \\
\hline Left & 65.5 & 21.9 & 23.5 & 82.4 & 94.8 \\
\hline Anti-Vax & 38.9 & 74.3 & 13.4 & 90.0 & 94.1 \\
\hline Right & 75.0 & 100.0 & 30.8 & 32.0 & 85.7 \\
\hline Neutral & 60.0 & 62.5 & 14.3 & 94.7 & 25.9 \\
\hline
\end{tabular}

Fig. 3 Ratio of targeted clusters and popular accounts in inter-cluster replies. a, Ratio of inter-cluster replies by source and target pairs in English; b, Ratio of popular accounts (with the number of followers $\geq 10,000$ ) in each targeted cluster. $\mathbf{c}$ and $\mathbf{d}$ are Japanese counterparts of $\mathbf{a}$ and $\mathbf{b}$, respectively.

these neutral accounts have a large number of followers, and in fact, the neutral accounts that received the most replies from anti-vaxxers were media and politicians' accounts. Contrarily, the percentage of replies from anti-vaxxer to pro-vaxxer groups was considerably small (shaded in yellow).

\section{Highly toxic replies by anti-vaxxers}

To characterise the nature of anti-vaxxers' inter-replies (i.e. replies to other groups), we measured the toxicity of languages used in replies (see Method) and tested its differences between inter- and inner-replies in each group (Figs. 4a and $4 \mathrm{~d}$ ). We found that the inter-cluster replies were significantly higher than the inner-cluster replies in both languages.

The toxicity of the inter-reply is compared between clusters in Figs. 4b and 4e. This reveals that the toxicity of the Anti-Vax's inter-reply is higher than the other groups, especially significantly higher than those of the Pro-Vax and Neutral groups in both English and Japanese ${ }^{9}$. Next, we examined who was more likely to receive a highly toxic reply from the Anti-Vax group. Looking at the toxicity of Anti-Vax's inter-cluster replies by target (see SI), it was found that the Anti-Vax's high toxicity is significantly directed at the Right cluster in English tweets. Upon scrutinisation of the content of the highly toxic replies from Anti-Vax to Right, we found that most of the replies were among the lines of 'Don't spread the vaccine'. As the Trump administration was in power, it seems that complaints and requests were made against the administration with high aggressiveness, even though the Right cluster was close to the Anti-Vax cluster in the RT network. On the contrary, in Japanese tweets, most of the toxic replies from Anti-Vax to Right were about criticism against

\footnotetext{
${ }^{9}$ On the other hand, we have to note that there were fewer tweets with very high toxicity overall (e.g., tweets with toxicity scores above $0.7[16,17]$ or $0.5[18]$ ), regardless of the cluster.
} 


\section{a}

\begin{tabular}{l|r|r|} 
Senders & \multicolumn{1}{|c|}{$\begin{array}{l}\text { inner- } \\
\text { cluster }\end{array}$} & \multicolumn{1}{l}{$\begin{array}{l}\text { inter- } \\
\text { cluster }\end{array}$} \\
\hline Pro-Vax & 0.09 & 0.092 \\
\hline Left & $0.109^{* * *}$ & 0.104 \\
\hline Anti-Vax & 0.109 & $0.112^{* * *}$ \\
\hline Right & 0.112 & 0.119 \\
\hline Neutral & 0.091 & 0.087
\end{tabular}

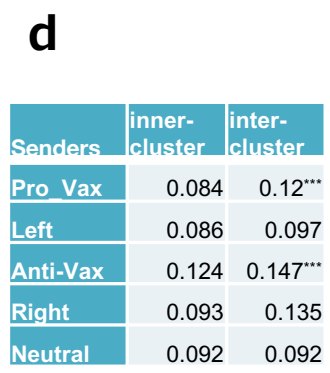

\section{English Tweet}

b
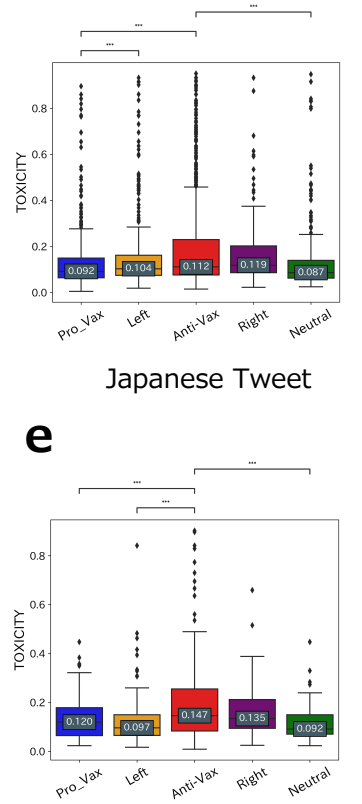

\section{$\mathbf{C}$}

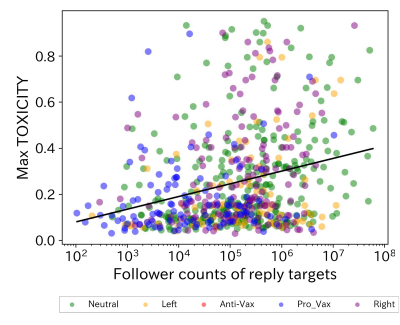

$\mathbf{f}$

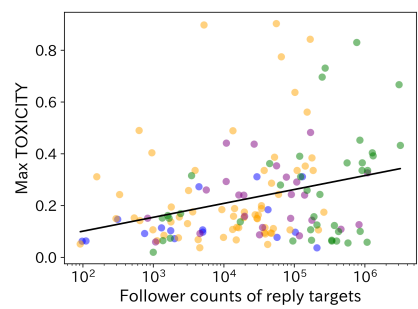

Fig. 4 Toxicity scores. a, Median toxicity scores of inner-cluster and inter-cluster replies from each cluster. Anti-Vax's inter-cluster replies have a significantly higher toxicity score than its inner-cluster replies. Left's inner-cluster replies have a significantly higher toxicity score than its inter-cluster replies. Both have $p<0.001$ by Mann-Whitney $\mathrm{U}$ test with Bonferroni correction. b, Boxplots representing the toxicity of the inter-cluster replies from each cluster. Each data point indicates the toxicity of a reply. Each median score is annotated in boxes. Replies from the Anti-Vax cluster were significantly more toxic than ones from the Pro-Vax and Neutral clusters in both languages $(* * * p<0.001$ by Mann-Whitney $U$ test with Bonferroni correction). c, The max toxicities that one received from Anti-Vax users. Each data point in the figure is the users who received the reply. The $\mathrm{x}$-axis is the number of followers of the users, and the y-axis is the toxicity of the replies received. The correlation coefficients are d: $0.02(p=0.601)$, e: $0.198(p \leq 0.001)$, h: $0.096(p=0.254)$, and 0.268 $(p \leq 0.005)$. $\mathbf{d}, \mathbf{e}$, and $\mathbf{f}$ are Japanese counterparts.

the administration party, although they did not concern the operation for vaccine rollout. The toxicity from Anti-Vax to Right clusters was not significantly high compared to those of other clusters.

Moreover, we found a positive correlation between toxicity and the number of followers of the target of Anti-Vax replies both in English and Japanese. Figs. 4c and 4f show the max toxicities one received from Anti-Vax users, indicating that users with a large number of followers are more likely to receive replies with high toxicity. In English, however, there are a certain number of accounts with a small number of followers that still receive highly toxic replies, and if we view them as less tolerant of toxicity than people with larger 

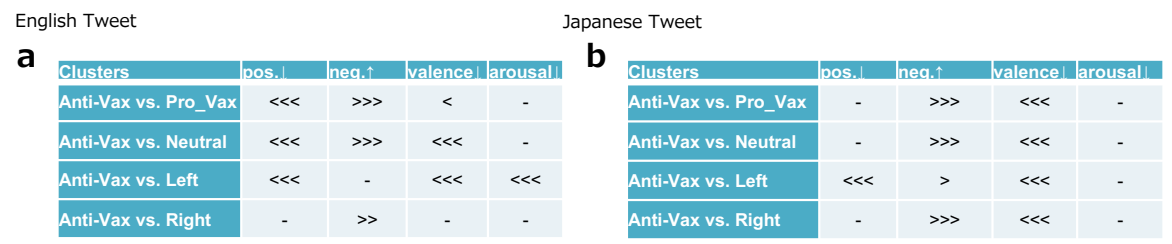

Fig. 5 Comparison of emotions of inter-cluster replies. a for English and $\mathbf{b}$ for Japanese. The comparison is based on each feature using the Mann-Whitney $U$ test with Bonferroni correction. The direction of brackets indicates the cluster with the larger amount. "<<<": $p<0.001, "<<": p<0.01, "<": p<0.05$.

followings, they should be careful about the toxic replies. We cannot find this tendency in Japanese tweets.

Finally, we evaluated the emotions of inter-cluster replies. Compared with the other clusters, replies from the Anti-Vax cluster contained more negative and less valence words in both English and Japanese, although the less use of positive words is apparent in English only (Fig. 5). This result is consistent with the finding that the Anti-Vax users' replies are toxic and thus negative.

\section{Discussion}

We have revealed that the replies of anti-vaxxers are highly active, targeted, and toxic compared to others' replies. As a consequence, the reply of antivaxxers can work as a targeted attack to influence vaccine belief and may provoke anxiety about vaccination in other clusters, because replies can reach beyond a follow-follower network. These characteristics are common in English and Japanese, although Western countries and Japan have different cultures and policies about vaccination. The repeated exposure to the same belief can affect people's perception efficiently, in what is known as the 'mere exposure effect' [19], coupled with subliminal techniques often used in propaganda [20, 21]. Furthermore, because their replies were directed to popular accounts such as news media, the possibility of being witnessed by users in other communities would be impactful. This kind of borrowing of the authority of prominent social media accounts is a typical strategy of spreading disinformation [22, 23]. Thus, a potential reason the anti-vaxxers actively send replies to neutral accounts (mostly media accounts) would be to attempt to propagate anti-vaccine beliefs and strengthen their influence.

Our study also showed that the inter-cluster replies of anti-vaxxers conveyed higher toxicity and negative sentiment. Previous research on effective vaccine narratives has shown that texts with strong emotionality were more likely to leave a greater impression on receivers than texts with a detailed description [24]. Other researchers have reported that influential users on social media tend to be individuals who express negative sentiments [25, 26]. Emotional messages effectively affect users who just witnessed the message even though they are not directed at them [27]. Considering this evidence, the toxic 
attack by the Anti-Vax is a potentially dangerous behaviour that may increase vaccine hesitancy and put anti-vaccine beliefs into other groups' minds.

Given the above characteristics of anti-vaxxers' replies that are common across languages, two important implications arise. First, platformers will be able to take measures to avoid exposing anti-vaccine discourse to other users. Reply behaviours can jump over the follow-follower relationships, and thus they can be exposed to the recipients and the followers of the recipients. Although reply behaviour cannot be banned due to freedom of expression, platformers can think about varying the priority of displaying replies or adding some delay, especially replies toward prominent accounts. The reply to prominent accounts is one place where we can see tweets with different beliefs. If there can be antivaccine-related replies, lowering the priority of displaying these replies would decrease the possibility of exposing them to other users.

Second, pro-vaxxers should prepare for toxic and emotional replies from anti-vaxxers. In the interviews with pro-vax organizations about their experiences with responding to anti-vaxxers [28], an interviewee highlighted the 'need to come across as the responsible, reasonable, calm ones because of all the people that are reading and not commenting'. This study also found that antivaxxers were negative in their expressions and tone. Those findings, including ours, should be shared by social media users as well as platformers. Although there are several attempts for fact-checking vaccine misinformation ${ }^{10}$, factchecking organisations should make more actionable guidelines to deal with high toxic replies from anti-vaxxers.

Equipped with countermeasures based on language-dependent and independent features of anti-vaxxers, such as ours, we can better guard from the toxic targeted attack by anti-vaxxers both at individual and platform levels.

\section{Methods}

\section{Data collection}

We collected COVID-19-related tweets using Twitter Search API from February to December 2020, which resulted in 8,579,728 English tweets and 1,952,376 Japanese tweets. The COVID-19-related words used for the search include 'corona virus', 'coronavirus', 'COVID19', '2019-nCoV', 'SARS-CoV-2', and 'wuhanpneumonia' and their counterparts in Japanese. We then extracted tweets that contained any of the vaccine-related words: 'vaccine', 'vax', and 'vaccination', and retained them for analysis. The resulting volume of tweets was $8,579,728$, of which $6,879,713(80.2 \%)$ were retweets (RT) and 293,946 $(3.43 \%)$ were replies (RP). The number of unique users was 2,799,034.

\section{Networks of anti-vaxxers and other groups}

We employed the RT network-based clustering to classify users according to their stance on vaccines. RT network clustering is a method of detecting users with similar stances by applying network clustering to an RT network [29,

\footnotetext{
${ }^{10}$ https://vaccinationdemandobservatory.org/
} 
30]. We used RT network clustering in our study for three reasons. First, on the topic of vaccines, research has shown that a network community can be easily divided by their stance on vaccines and emerges echo chambers $[8$, 31], which led us to believe that we could get anti-vaccine clusters using this method. Second, RT network clustering can automatically reveal clusters of like-minded users without imposing any thresholds for classification. Last, it does not present the problem with an arbitrariness that differentiating pro-vax from anti-vax hashtags does.

To construct the RT network, using all data from February to December 2020, we created an edge between users with more than two RTs (including mutual RTs). As a result, the meaning of the endorsement was more robustly incorporated into the edges [32]. After creating the network, we applied $k$ core decomposition $(k=3)$ to exclude users with only weak connections to the primary discussions [33]. Next, the Louvain method was used to cluster anti-vaccine users and other groups [34]. Due to the constraints RT $\geq 2$ and $k$ core $=3$, the number of users was reduced to only those participating in the discussion about vaccines.

We found the five clusters by the aforementioned method and named each cluster by looking at their retweeted texts (see the word clouds in Fig. 1 and raw texts $(\mathrm{SI})$ ) and the representative accounts that were most retweeted.

\section{Measurement of toxicity}

To measure the degree of attack conveyed by tweets toward others on Twitter, we employed Google's Perspective API ${ }^{11}$, a popular tool widely used for online abuse and harassment study $[16,17,35]$. The perspective API allows users to measure the toxicity of a text in English on a scale from 0 to 1 . For measuring the toxicity from the text in Japanese, we needed to translate it to English, as the perspective API is only available in English. To this end, we used the Google Translate translator $\mathrm{API}^{12}[36]$.

\section{Measurement of emotions}

To evaluate the emotions of replies, we adopted an approach of counting the words registered in the sentiment dictionary. As for the positive and negative emotions, we used LIWC 2015 dictionary [37]. As for the level of arousal and valence, we used the dictionary of Warriner, Kuperman, and Brysbaert [38]. We counted the number of words per tweet with scores above the median for each metric in the dictionary because the dictionary also contains words with low scores. In measuring emotions, we translated Japanese tweets into English using the Google Translate translator API, the same as measuring toxicity.

\section{Data availability}

The tweet IDs used in this study will be available when the manuscript is accepted.

\footnotetext{
${ }^{11}$ https://www.perspectiveapi.com/

12 https://cloud.google.com/translate/
} 


\section{Code availability}

The code used for analysis will be available when the manuscript is accepted. The open-source software Gephi and Python libraries were used to compute statistics and produce the figures.

\section{Acknowledgements}

This work was supported by JST, CREST Grant Number JPMJCR20D3, Japan.

\section{References}

[1] Agley, J., Xiao, Y.: Misinformation about covid-19: evidence for differential latent profiles and a strong association with trust in science. BMC Public Health 21(1), 1-12 (2021)

[2] Burki, T.: Vaccine misinformation and social media. The Lancet Digital Health 1(6), 258-259 (2019)

[3] Fontanet, A., Cauchemez, S.: Covid-19 herd immunity: where are we? Nature Reviews Immunology 20(10), 583-584 (2020)

[4] Kata, A.: Anti-vaccine activists, web 2.0, and the postmodern paradigman overview of tactics and tropes used online by the anti-vaccination movement. Vaccine 30(25), 3778-3789 (2012)

[5] Brennen, J.S., Simon, F., Howard, P.N., Nielsen, R.K.: Types, sources, and claims of covid-19 misinformation. Reuters Institute $7(3), 1$ (2020)

[6] Germani, F., Biller-Andorno, N.: The anti-vaccination infodemic on social media: A behavioral analysis. PloS ONE 16(3), 0247642 (2021)

[7] Mitra, T., Counts, S., Pennebaker, J.: Understanding anti-vaccination attitudes in social media. In: Proceedings of the International AAAI Conference on Web and Social Media, vol. 10 (2016)

[8] Gunaratne, K., Coomes, E.A., Haghbayan, H.: Temporal trends in antivaccine discourse on twitter. Vaccine 37(35), 4867-4871 (2019)

[9] Apuke, O.D., Omar, B.: Fake news and covid-19: modelling the predictors of fake news sharing among social media users. Telematics and Informatics 56, 101475 (2021)

[10] Sasahara, K., Chen, W., Peng, H., Ciampaglia, G.L., Flammini, A., Menczer, F.: Social influence and unfollowing accelerate the emergence of echo chambers. Journal of Computational Social Science, 1-22 (2020) 
[11] Choi, D., Chun, S., Oh, H., Han, J., et al.: Rumor propagation is amplified by echo chambers in social media. Scientific reports 10(1), 1-10 (2020)

[12] Sharun, K., Dhama, K.: Covid-19 vaccine diplomacy and equitable access to vaccines amid ongoing pandemic. Archives of Medical Research (2021)

[13] MAA, A.M.J.: Vaccine communication as weaponized identity politics. American journal of public health 110(5), 617-618 (2020)

[14] Broniatowski, D.A., Jamison, A.M., Qi, S., AlKulaib, L., Chen, T., Benton, A., Quinn, S.C., Dredze, M.: Weaponized health communication: Twitter bots and russian trolls amplify the vaccine debate. American Journal of Public Health 108(10), 1378-1384 (2018)

[15] Johnson, N.F., Velásquez, N., Restrepo, N.J., Leahy, R., Gabriel, N., El Oud, S., Zheng, M., Manrique, P., Wuchty, S., Lupu, Y.: The online competition between pro-and anti-vaccination views. Nature 582(7811), 230-233 (2020)

[16] Hua, Y., Naaman, M., Ristenpart, T.: Characterizing twitter users who engage in adversarial interactions against political candidates. In: Proceedings of the 2020 CHI Conference on Human Factors in Computing Systems, pp. 1-13 (2020)

[17] Hua, Y., Ristenpart, T., Naaman, M.: Towards measuring adversarial twitter interactions against candidates in the us midterm elections. In: Proceedings of the International AAAI Conference on Web and Social Media, vol. 14, pp. 272-282 (2020)

[18] Gehman, S., Gururangan, S., Sap, M., Choi, Y., Smith, N.A.: Realtoxicityprompts: Evaluating neural toxic degeneration in language models. In: Findings of the Association for Computational Linguistics: EMNLP 2020, pp. 3356-3369 (2020)

[19] Bornstein, R.F.: Exposure and affect: Overview and meta-analysis of research, 1968-1987. Psychological Bulletin 106(2), 265 (1989)

[20] Bornstein, R.F., D'agostino, P.R.: Stimulus recognition and the mere exposure effect. Journal of Personality and Social Psychology 63(4), 545 (1992)

[21] Bornstein, R.F.: Subliminal techniques as propaganda tools: Review and critique. The Journal of Mind and Behavior, 231-262 (1989)

[22] Benkler, Y., Faris, R., Roberts, H.: Network Propaganda: Manipulation, Disinformation, and Radicalization in American Politics. Oxford University Press, ??? (2018) 
[23] Watts, D.J., Rothschild, D.M., Mobius, M.: Measuring the news and its impact on democracy. Proceedings of the National Academy of Sciences 118(15) (2021)

[24] Betsch, C., Ulshöfer, C., Renkewitz, F., Betsch, T.: The influence of narrative $\mathrm{v}$. statistical information on perceiving vaccination risks. Medical Decision Making 31(5), 742-753 (2011)

[25] Quercia, D., Ellis, J., Capra, L., Crowcroft, J.: In the mood for being influential on twitter. In: 2011 IEEE Third International Conference on Privacy, Security, Risk and Trust and 2011 IEEE Third International Conference on Social Computing, pp. 307-314 (2011). IEEE

[26] Xiao, L., Khazaei, T.: Changing others' beliefs online: Online comments' persuasiveness. In: Proceedings of the 10th International Conference on Social Media and Society, pp. 92-101 (2019)

[27] Grandjean, D., Sander, D., Pourtois, G., Schwartz, S., Seghier, M.L., Scherer, K.R., Vuilleumier, P.: The voices of wrath: brain responses to angry prosody in meaningless speech. Nature neuroscience 8(2), 145-146 (2005)

[28] Steffens, M.S., Dunn, A.G., Wiley, K.E., Leask, J.: How organisations promoting vaccination respond to misinformation on social media: a qualitative investigation. BMC Public Health 19(1), 1-12 (2019)

[29] Fortunato, S.: Community detection in graphs. Physics Reports 486(3-5), 75-174 (2010)

[30] Conover, M., Ratkiewicz, J., Francisco, M., Gonçalves, B., Menczer, F., Flammini, A.: Political polarization on twitter. In: Proceedings of the International AAAI Conference on Web and Social Media, vol. 5 (2011)

[31] Cossard, A., Morales, G.D.F., Kalimeri, K., Mejova, Y., Paolotti, D., Starnini, M.: Falling into the echo chamber: the italian vaccination debate on twitter. In: Proceedings of the International AAAI Conference on Web and Social Media, vol. 14, pp. 130-140 (2020)

[32] Garimella, K., Morales, G.D.F., Gionis, A., Mathioudakis, M.: Quantifying controversy on social media. ACM Transactions on Social Computing 1(1), 1-27 (2018)

[33] Alvarez-Hamelin, J.I., Dall'Asta, L., Barrat, A., Vespignani, A.: Large scale networks fingerprinting and visualization using the k-core decomposition. In: Advances in Neural Information Processing Systems, pp. 41-50 (2006) 
[34] Blondel, V.D., Guillaume, J.-L., Lambiotte, R., Lefebvre, E.: Fast unfolding of communities in large networks. Journal of Statistical Mechanics: Theory and Experiment 2008(10), 10008 (2008)

[35] Wu, S., Resnick, P.: Cross-partisan discussions on youtube: Conservatives talk to liberals but liberals don't talk to conservatives 15, (2021)

[36] Samoilenko, A., Lemmerich, F., Weller, K., Zens, M., Strohmaier, M.: Analysing timelines of national histories across wikipedia editions: A comparative computational approach. In: Proceedings of the International AAAI Conference on Web and Social Media, vol. 11 (2017)

[37] Pennebaker, J.W., Boyd, R.L., Jordan, K., Blackburn, K.: The development and psychometric properties of liwc2015. Technical report (2015)

[38] Warriner, A.B., Kuperman, V., Brysbaert, M.: Norms of valence, arousal, and dominance for 13,915 english lemmas. Behavior Research Methods 45(4), 1191-1207 (2013) 


\section{Supplementary Files}

This is a list of supplementary files associated with this preprint. Click to download.

- NatureHumanBehavior2021draft215.pdf 